\title{
Development and Validation of Hub Genes for Adrenal Aldosterone-Producing Adenoma by Integrated Bioinformatics Analysis
}

\author{
Hai Cai ${ }^{1, *}$ \\ Shao-Ming Chen ${ }^{2, *}$ \\ Zhi-Bin $\mathrm{Ke}^{\mathrm{l}, *}$ \\ Hang Chen ${ }^{\mathrm{l}, *}$ \\ Jun-Ming Zhu' \\ Ting-Ting Lin' \\ Fei Huang ${ }^{3,4}$ \\ Yong Wei id \\ Qing-Shui Zheng' \\ Xue-Yi Xue (D) \\ Xiong-Lin Sun ${ }^{1}$ \\ Ning $X u$ iD $^{1,3}$

\begin{abstract}
'Department of Urology, Urology Research Institute, The First Affiliated Hospital, Fujian Medical University, Fuzhou, 350005, People's Republic of China; ${ }^{2}$ Department of Nuclear Medicine, First Affiliated Hospital of Fujian Medical University, Fuzhou, 350005, People's Republic of China; ${ }^{3}$ Fujian Key Laboratory of Precision Medicine for Cancer, The First Affiliated Hospital, Fujian Medical University, Fuzhou, 350005, People's Republic of China; ${ }^{4}$ Central Lab, The First Affiliated Hospital of Fujian Medical University, Fuzhou, 350005, People's Republic of China

*These authors contributed equally to this work
\end{abstract}

Correspondence: Ning Xu; Xiong-Lin Sun Tel +86-059187981687

Email drxun@fjmu.edu.cn; sunxionglin@fjmu.edu.cn
Objective: To develop and validate hub genes involving in the development and progression of primary aldosteronism (PA) and adrenal aldosterone-producing adenoma (APA).

Materials and Methods: A total of four datasets of gene expression profiles related to APA were downloaded from GEO datasets. GSE60042 and GSE8514 were used to identify DEGs. Weighted gene co-expression network analysis (WGCNA) and protein-protein interaction (PPI) network module analysis were conducted. GO and KEGG enrichment analysis was performed. GSE10927 and GSE33371 were used for further external validation.

Results: We identified a total of 892 DEGs from GSE60042 and 1167 DEGs from GSE8514. WGCNA analysis demonstrated that the blue module (255 genes) and turquoise module (303 genes) were significantly correlated with APA. PPI networks were then constructed. GO term enrichment analysis suggested that cellular divalent inorganic cation homeostasis, calcium ion homeostasis, collagen-containing extracellular matrix, transport vesicle and metal ion transmembrane transporter activity were the vital annotations. KEGG pathway analysis found that these genes were significantly enriched in neuroactive ligand-receptor interaction, calcium signaling pathway. Finally, we identified a total of 11 candidate genes involving in the development and progression of APA and PA. Besides, two independent datasets (GSE10927 and GSE33371) were used for external validation, and there were seven hub genes successfully verified, including C3, GRM3, AVPR1A, WFS1, PTGFR, NTSR2, and JUN.

Conclusion: These newly identified genes could contribute to the understanding of potential mechanism in APA and PA and might be promising targets for the treatment of APA and PA. Keywords: aldosterone-producing adenoma, bioinformatics, primary aldosteronism, gene expression omnibus datasets

\section{Background}

Primary hyperaldosteronism (PA) is characterized by spontaneous secretion of excessive aldosterone and inhibition of plasma renin activity. ${ }^{1}$ The pathogenesis of adrenal aldosterone-producing adenoma (APA) involves the abnormal proliferation of adrenal cortex cells and the excessive secretion of aldosterone, accounting for nearly $30 \%$ of PA. Excessive secretion of aldosterone can lead to retention of sodium, suppression of plasma renin levels, increased excretion of potassium, secondary hypertension (HTN) and end-organ damage. ${ }^{2,3}$ APA is known as a heterogeneous disease. The occurrence, development and metastasis of tumors are usually attributed to the combined effects of genetic aberrations, cell environment changes and internal and external environmental influences. Therefore, 
clarifying the cause, molecular mechanisms and key pathways of carcinogenesis of APA is of great significance for improving the diagnosis and treatment of APA.

At present, there are few studies focusing on comprehensive analysis of changes in mRNA expression involving the development of APA and PA. Previous studies have discovered several new genes in APA, but there are some limitations that cannot be ignored. Recently, weighted correlation network analysis (WGCNA) is considered to be an effective method for processing gene expression data and discovering new key genes linked to the clinicopathological characteristics of interest. ${ }^{4,5}$ According to the pairwise correlation, highly coexpressed genes can constitute multiple gene modules. Then, the genes enriched in the identified modules are screened for further analysis. Therefore, WGCNA has been widely used to determine indicators in various cancers, including glioblastoma, ${ }^{4}$ bladder cancer,${ }^{6}$ breast cancer, ${ }^{7}$ and adrenocortical carcinoma. ${ }^{8}$ However, there have been no previous studies using WGCNA to study the underlying mechanism of APA and PA.

In the present study, we downloaded the gene expression profiles of GSE60042 and GSE8514 from the Gene Expression Collection (GEO) dataset. ${ }^{9,10}$ All APA samples were taken from patients with adrenal adenoma. We compared the gene expression profiles of APA samples and normal adrenal gland samples to identify differentially expressed genes (DEGs). WGCNA was performed to screen key modules and genes. Further bioinformatics analysis was used to clarify potential annotations and pathways of APA and PA. Protein-protein interaction (PPI) network module analysis was also constructed. Finally, the gene expression profiles of GSE10927 and GSE33371 were used for further validation. ${ }^{11,12}$

\section{Materials and Methods}

\section{Microarray Data}

A total of four datasets of gene expression profiles related to aldosterone-producing adenoma (including GSE60042, GSE8514, GSE10927 and GSE33371) were downloaded from Gene Expression Omnibus (GEO) datasets (https:// www.ncbi.nlm.nih.gov/geo/). There were 7 samples of APA and 7 samples of adjacent adrenal tissue in GSE60042, 10 samples of APA and 5 samples of normal adrenal tissue in GSE8514. R packages "limma" was used to identify DEGs between APA and normal adrenal tissue in
GSE60042 and GSE8514. We set false discovery rate (FDR) $<0.05$ and $\mid \log$ fold change (FC) $\mid \geq 1$ as the cut-off criterion.

\section{Weighted Gene Co-Expression Network Analysis (WGCNA) of DEGs}

"WGCNA" package in R software was used to perform WGCNA. Firstly, data pre-processing and quality assessment were performed, and the Pearson's correlation matrices were constructed for all paired genes. Furthermore, a weighted adjacency matrix was established utilizing a power function $\mathrm{a}_{\mathrm{mn}}=\left|\mathrm{c}_{\mathrm{mn}}\right|^{\beta}\left(\mathrm{c}_{\mathrm{mn}}=\right.$ Pearson's correlation between gene $m$ and gene $n ; a_{m n}$ = adjacency between gene $\mathrm{m}$ and gene $\mathrm{n}$ ). The parameter $\beta$ emphasized a strong correlation between genes and penalized a weak correlation. We selected an applicable $\beta$ value to increase the similarity matrix and achieve a scale-free co-expression network. Next, we converted the adjacency matrix into a topological overlap matrix (TOM). Gene significance (GS) was defined as the $\log 10$ transformation of the P-value (GS $=\operatorname{lgP})$ in the linear regression between gene expression and the clinical features. Module significance (MS) was defined as the average GS within the module. Module eigengene (ME) was considered to be the major component in the principal component analysis for each gene module. We explored the relationship between clinical features and ME to filtrate modules. Finally, the genes in module highly related to aldosterone-producing adenoma were selected for subsequent analysis.

\section{Protein-Protein Interaction (PPI) Network Construction}

Search Tool for the Retrieval of Interacting Genes (STRING, version 11.0, https://string-db.org/cgi/input.pl) database was used to evaluate the protein-protein interaction (PPI) information. The genes were mapped in STRING database, and interactive relationships were then produced and downloaded. PPI networks were then visualized by using the Cytoscape software. The plug-in "CentiScape" was used to calculate centrality parameters for screen the most important nodes. Degree score $>5$ was set as the criteria. The plug-in Molecular Complex Detection (MCODE) was used to screen the top key modules in PPI network.

\section{Functional Enrichment Analyses}

Functional enrichment analyses, including Gene Ontology (GO) analysis and the Kyoto Encyclopedia of Genes and 
Genomes (KEGG) pathway analysis, were performed using $\mathrm{R}$ package "clusterProfiler", “org.Hs.eg.db", "enrichplot" and "ggplot2". The results of GO analysis included biological processes (BP), molecular functions (MF), and cellular component (CC).

\section{Validation of Identified Intersection Genes}

In order to further verify the results, two independent datasets, including GSE10927 and GSE33371, were used as an external validation. There were 6 samples of APA and 10 samples of adjacent adrenal tissue in GSE10927, 8 samples of APA and 10 samples of normal adrenal tissue in GSE33371. Wilcoxon test and $\mathrm{R}$ packages "limma" was used to compare the expression levels of these seven hub genes between APA tissues and normal adrenal tissues. $\mathrm{P}<0.05$ was considered statistically significant.

\section{Results}

\section{Identification of DEGs in GSE60042}

By comparing APA samples and adjacent adrenal tissue in GSE60042, a total of 892 DEGs were identified for the subsequent analysis, consisting of 569 significantly downregulated DEGs and 323 significantly upregulated DEGs. The volcano plot and heatmap of these DEGs are demonstrated in Figure 1. All the 892 DEGs were extracted for WGCNA analysis.

A

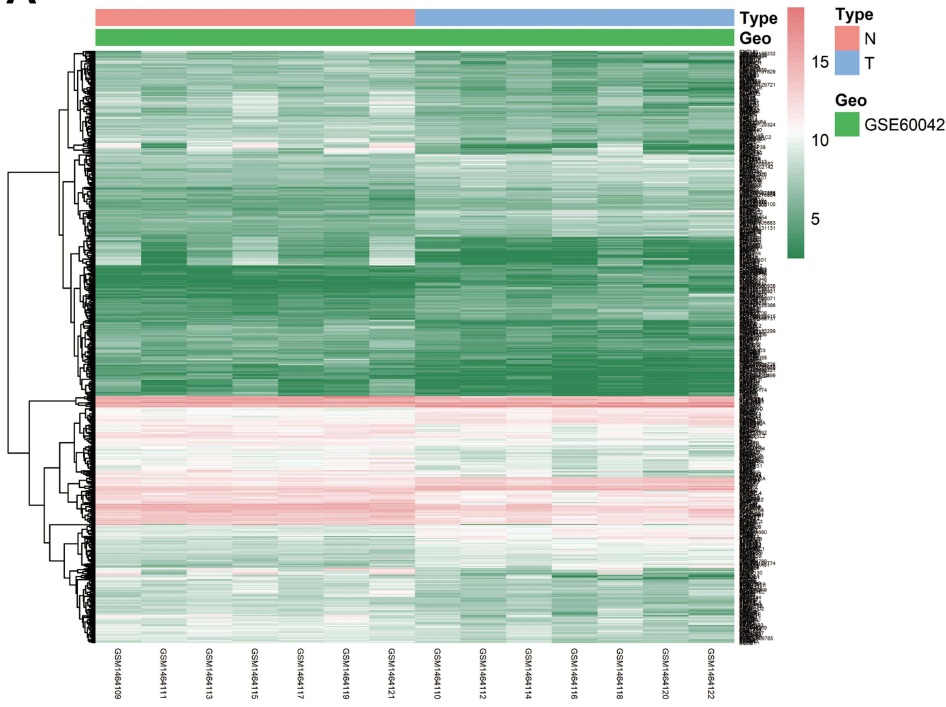

Weighted Gene Co-Expression Network Analysis DEGs from GSE60042

WGCNA was applied to identify stable co-expression modules related to APA. Cluster analysis and dynamic tree cut algorithm were performed to generate six gene modules, including blue, brown, green, grey, turquoise and yellow. Each color represented one gene module. Each gene module consisted of a number of similar gene expression patterns. Finally, we found that the blue module has the most positive correlation with APA, while the turquoise module has the most negative correlation with APA. A total of 558 genes (including 255 genes in blue module and 303 genes in turquoise module) were considered to be most relevant to APA in Figure 2.

Next, we mapped these 558 genes identified from WGCNA into STRING database to construct PPI network. The PPI network consisted of 396 nodes and 923 edges. Using plug-in "CentiScape" with degree of connectivity $>5$ as selection criteria, we eventually identify a total of 134 genes of great importance in APA development.

\section{Functional Enrichment Analyses}

GO term enrichment analysis results varied from GO classification and expression change of DEGs. As to biological process, the DEGs significantly enriched in cellular divalent inorganic cation homeostasis, calcium ion homeostasis, heart contraction and heart process. For cellular component, the DEGs significantly enriched in collagen -containing extracellular matrix, transport vesicle and transport vesicle membrane. About molecular function,

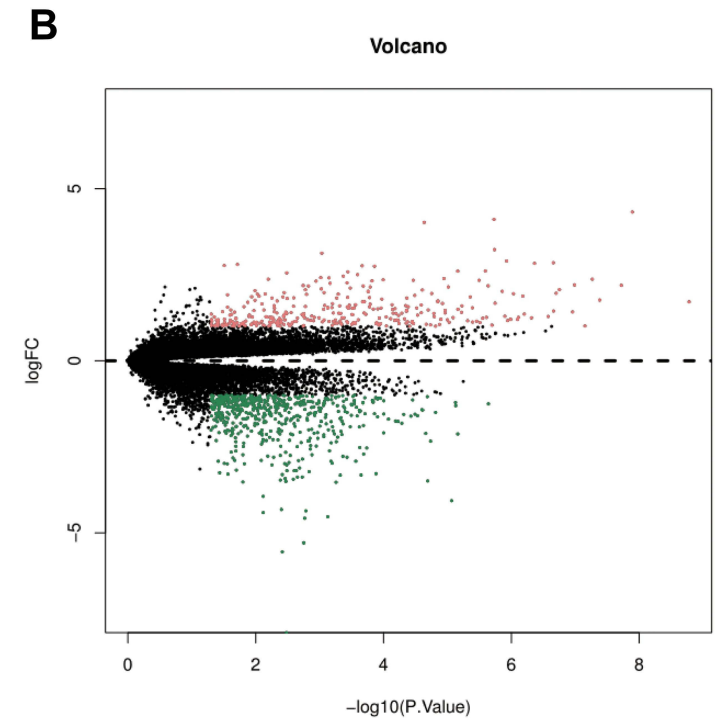

Figure I (A) Heatmap and (B) Volcano plot of differentially expressed genes from GSE60042. Red represents upregulated differentially expressed genes; green represents downregulated differentially expressed genes. 
A

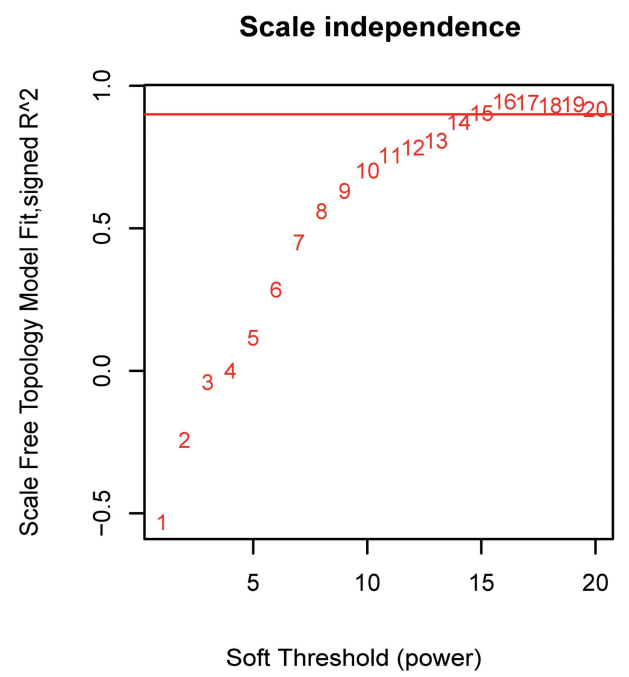

C

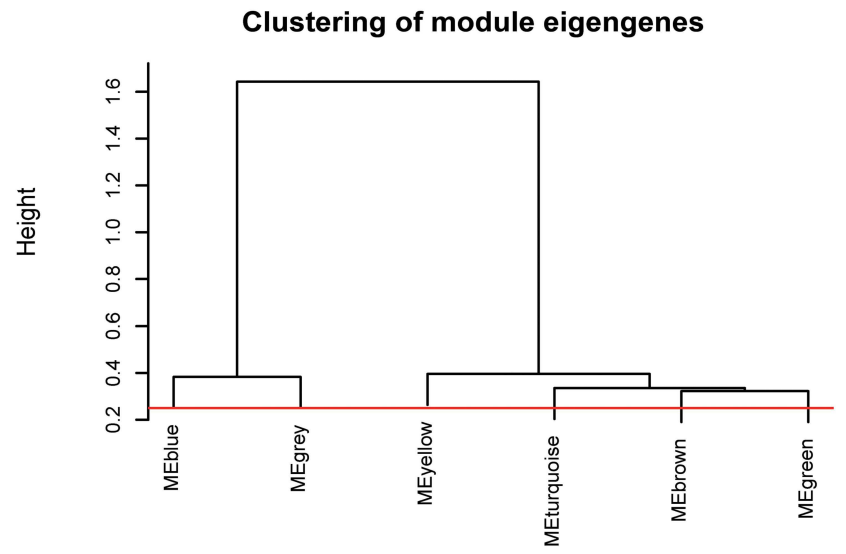

D

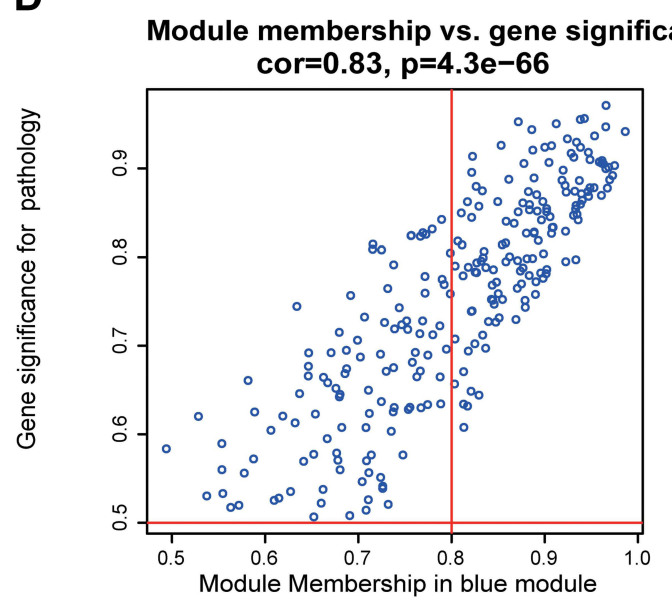

B

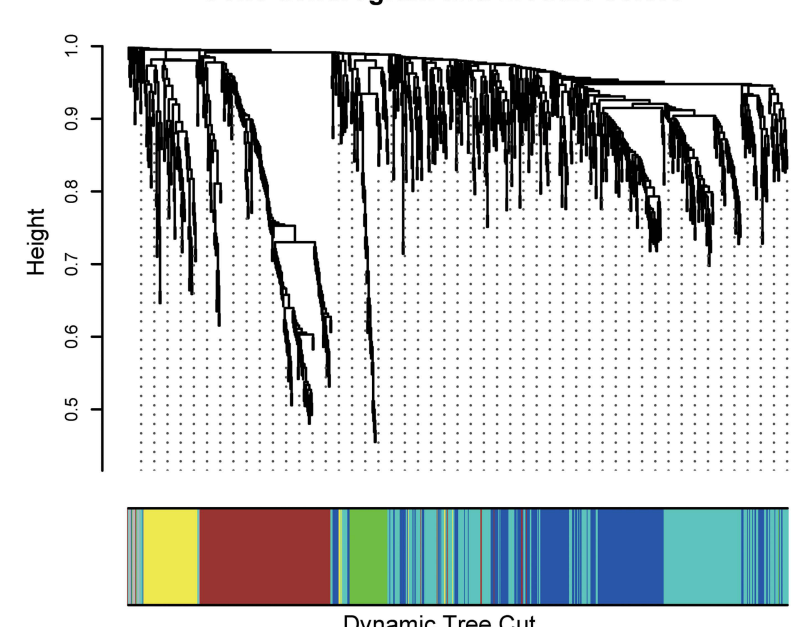

Gene dendrogram and module colors

Dynamic Tree Cut

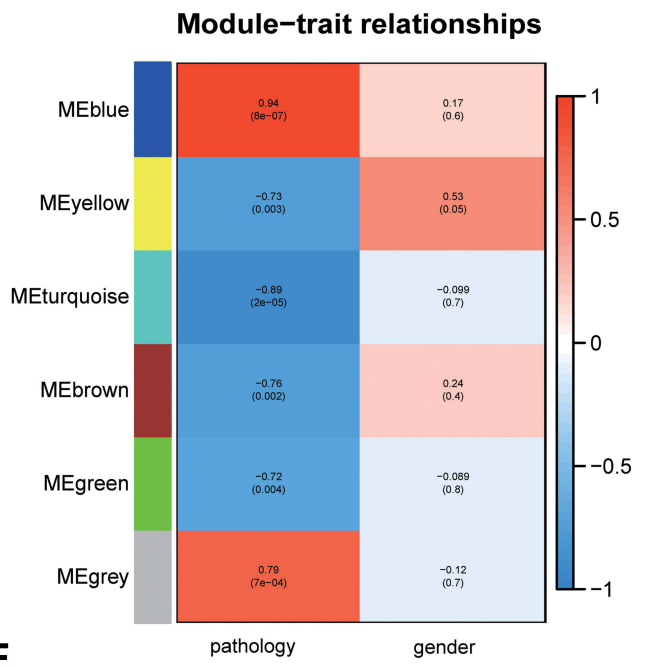

E

Module membership vs. gene significance cor $=0.79, p=6.5 \mathrm{e}-66$

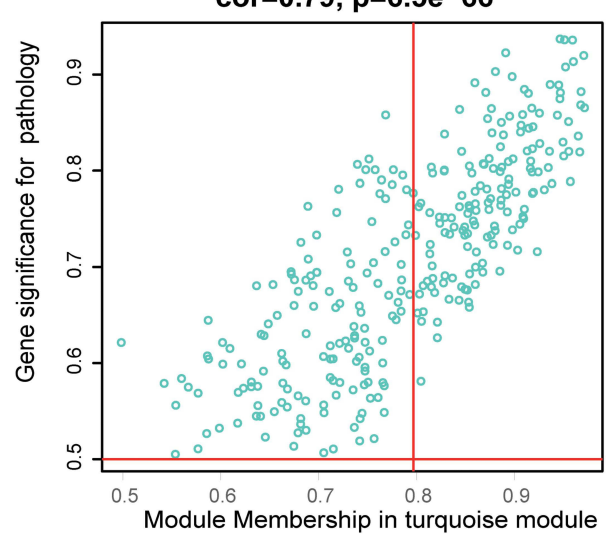

Figure 2 The results of WGCNA. (A) The calculation diagram of the weight parameter (power) of the adjacency matrix. The Xaxis represents the weight parameter (power). The Yaxis represents the square of the correlation index from $\log (K)$ and $\log (P(K))$. (B) WGCNA reveals clustering and modular screening based on gene expression patterns. The top is the gene tree diagram, and the bottom is the gene modules in different colors. (C) Dendrogram of characteristic genes of consensus modules obtained by WGCNA. The red line is the merging threshold. Feature genomes below the threshold represent the modules whose expression profiles should be merged due to their similarity. Clarified the overlap between the modules. Each row and each column corresponds to a module. The colors in the table indicate the gene counts at the intersection of the corresponding modules. (D) The correlation between MEblue membership and gene significance. (E) Correlation between MEturquise membership and gene significance. 
A

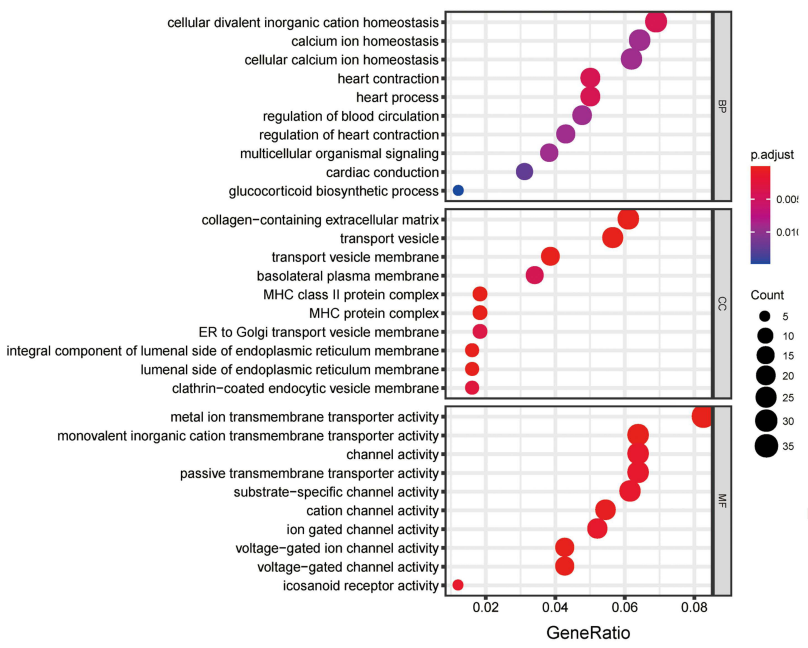

B

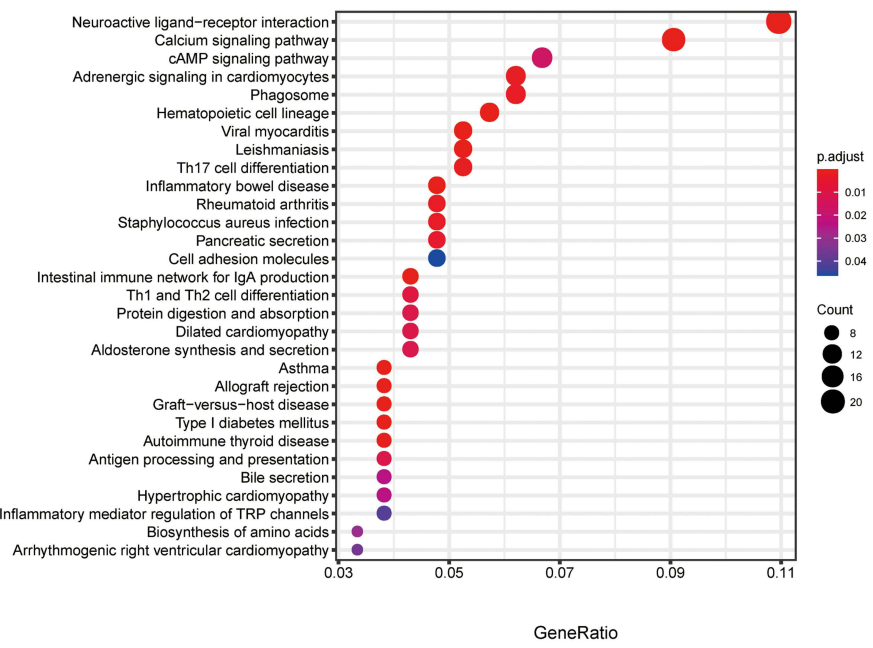

Figure 3 (A) GO and (B) KEGG enrichment analysis of 558 genes (including 255 genes in blue module and 303 genes in turquoise module) identified from WGCNA.

the DEGs significantly enriched in metal ion transmembrane transporter activity, monovalent inorganic cation transmembrane transporter activity, channel activity, passive transmembrane transporter activity, substrate-specific channel activity and cation channel activity. More detailed GO enrichment analysis results are shown in Figure 3A. KEGG pathway analysis found five significantly enriched pathways. The DEGs significantly enriched in neuroactive ligand-receptor interaction, calcium signaling pathway, adrenergic signaling in cardiomyocytes, phagosome, hematopoietic cell lineage. More detailed KEGG pathway analysis results are shown in Figure 3B. These significantly enriched pathways and terms could help us a lot to further understand the role which DEGs played in APA occurrence and progress.

\section{Identification of DEGs in GSE85/4}

By comparing APA samples and adjacent adrenal tissue in GSE8514, we screened a total of 1167 DEGs including 559 down-regulated DEGs and 608 upregulated DEGs. The volcano plot and heatmap of these DEGs in GSE8514 are demonstrated in Figure 4. All the 1167 DEGs were extracted for further PPI network construction.
A

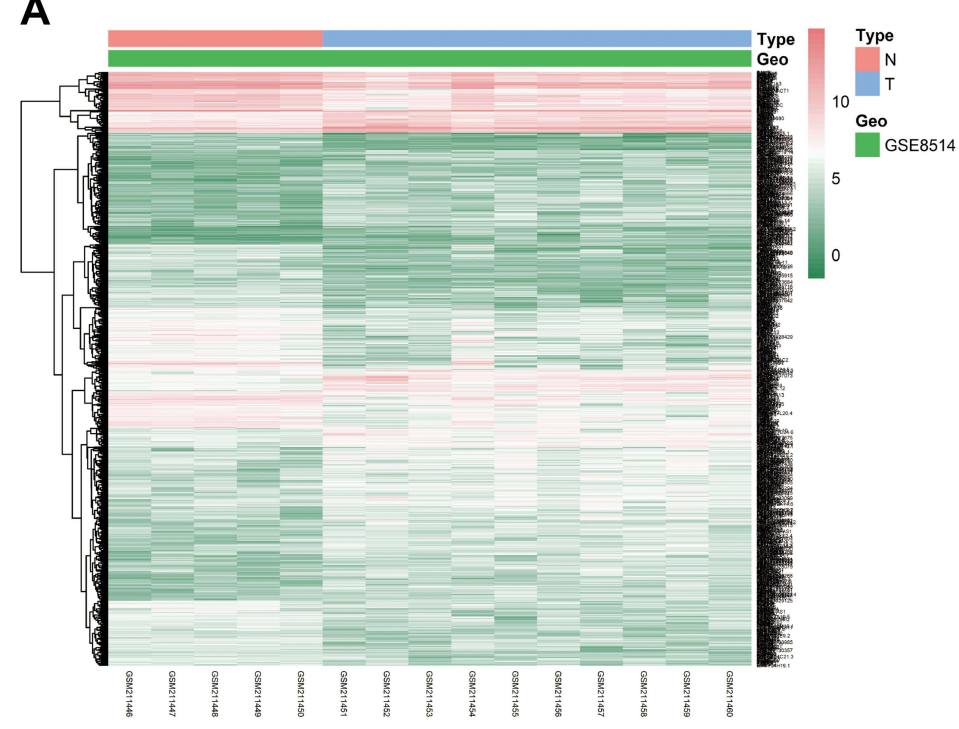

B

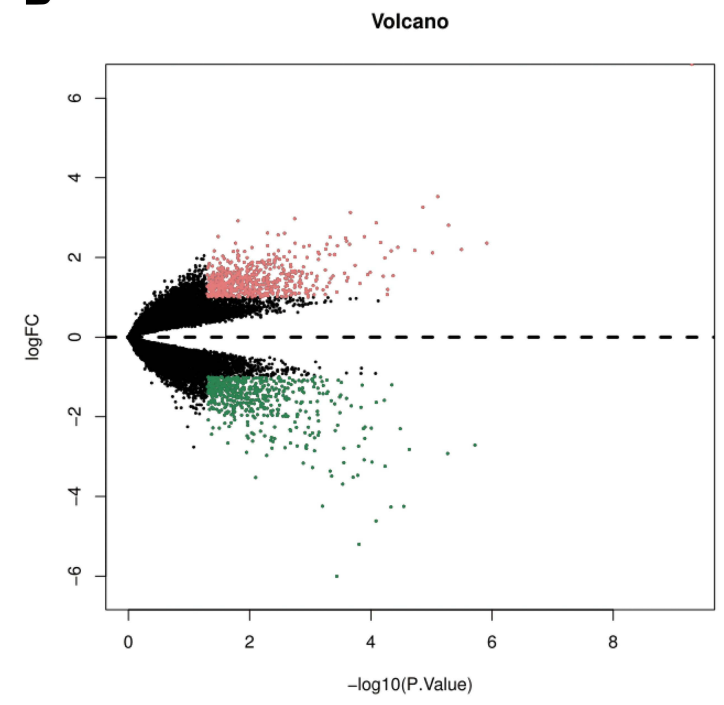

Figure 4 (A) Heatmap and (B) Volcano plot of differentially expressed genes from GSE85I4. Red represents upregulated differentially expressed genes; green represents downregulated differentially expressed genes. 


\section{Protein-Protein Interaction Network Analysis of DEGs from GSE85 I4}

The relationship between protein-protein interactions (PPI) was downloaded from the STRING database, consisting of 720 nodes and 2781 edges. Using plug-in MCODE, we identify the two most significant module from the PPI networks. The first module has 17 hub genes, including GPER1, TAS2R5, ACKR3, S1PR4, CCL4, NPY, HCAR1, GRM3, HTR5A, PENK, SST, CORT, CHRM4, CXCL12, CCL21, TAS2R10, C3 in Figure 5A. The second module has 37 hub genes, including SOCS3, CSF2, CCND1, JUN, PTPRC, IL1R1, IL10, PTGS2, FOS, NOD2, CD19, CD40LG, PPARG, CCL2, LEP, ADIPOQ, IL6, WFS1, IGFBP1, SCG3, FGA, SCG2, FGF23, FGG, CHRDL1, CHGB, CCKBR, AVPR1A, PTGFR, TAC1, PLCB4, CCK, NTS, NTSR2, TACR2, LPAR4, NMBR in Figure 5B.

\section{Identification of Intersection Genes}

Finally, we identify 134 genes from GSE60042 and 54 genes from GSE8514 as the candidate genes for APA. The Venn diagram showed that there were 11 intersection genes, including CCL21, CXCL12, C3, GRM3, AVPR1A, IGFBP1, WFS1, PTGFR, NTSR2, CCK, JUN in Figure 5C. These 11 genes were considered as the hub genes involved in the development and progression of primary aldosteronism (PA) and aldosterone-producing adenoma.

\section{Validation of Hub Genes in Two Independent Cohort}

Moreover, we utilized two independent datasets (GSE10927 and GSE33371) to validate the differential expression of these intersection genes between APA tissues and normal adrenal tissues. Eventually, a total of seven genes were successfully verified, including C3, GRM3, AVPR1A, WFS1, PTGFR, NTSR2, JUN. The expression levels of these seven hub genes between APA tissues and normal adrenal tissues from GSE10927 and GSE33371 were demonstrated in Figures 6 and 7, respectively.

\section{Discussion}

PA is the most common cause of secondary hypertension and accounts for approximately $20 \%$ of resistant hypertension. ${ }^{13}$ It has been known to us that bilateral
A

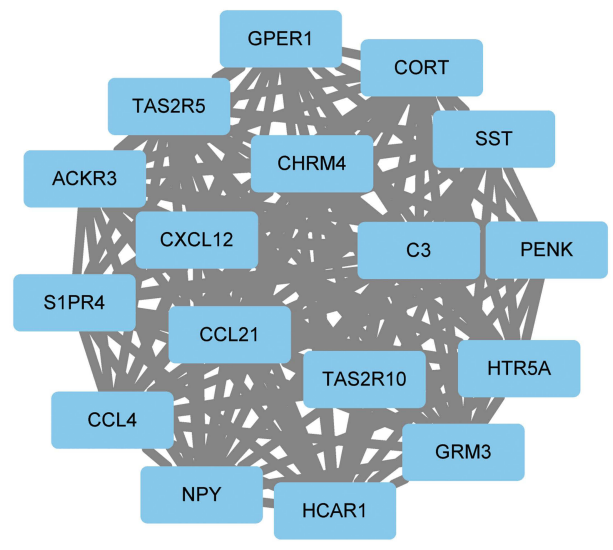

B

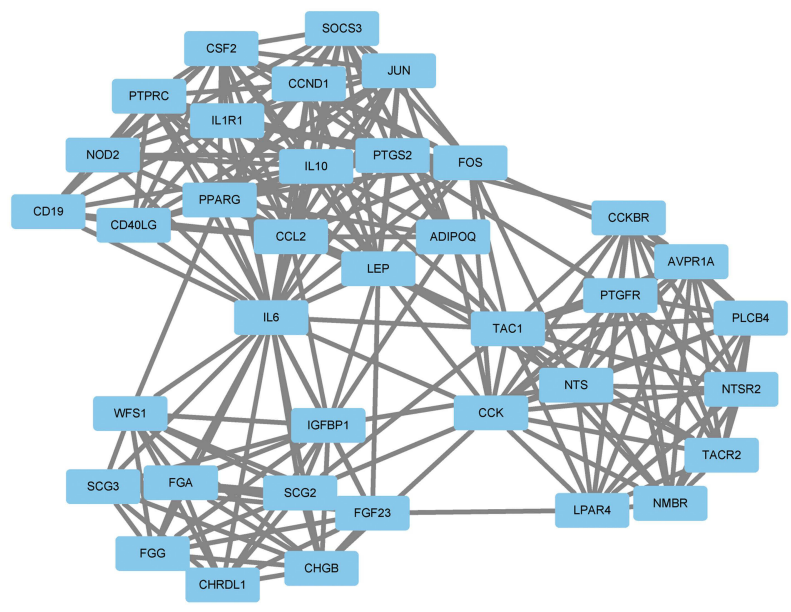

C

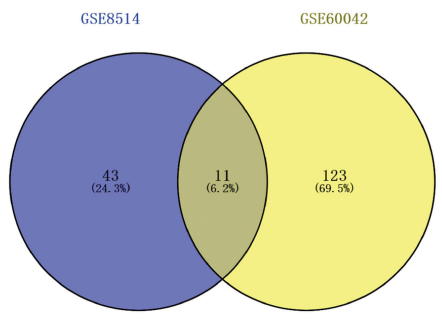

Figure 5 Two most significant module in the protein-protein interaction (PPI) network analysis (A) First module in the PPI network. (B) Second module in the PPI network. (C) Venn diagram used to identify cross genes. 
A

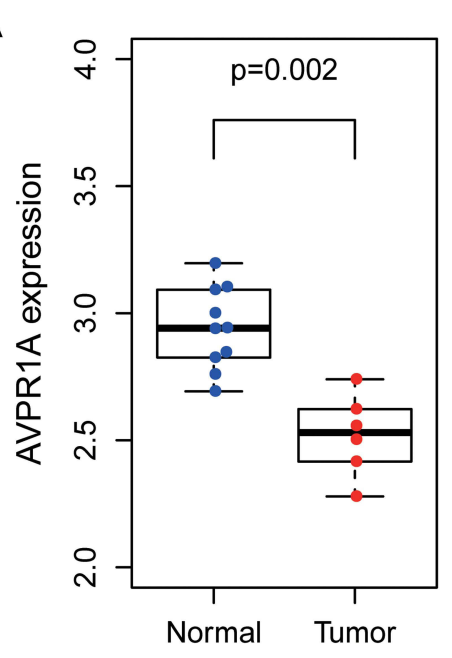

D

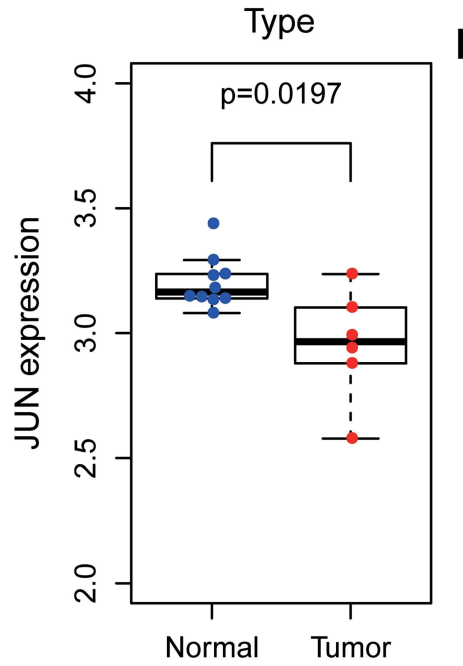

G

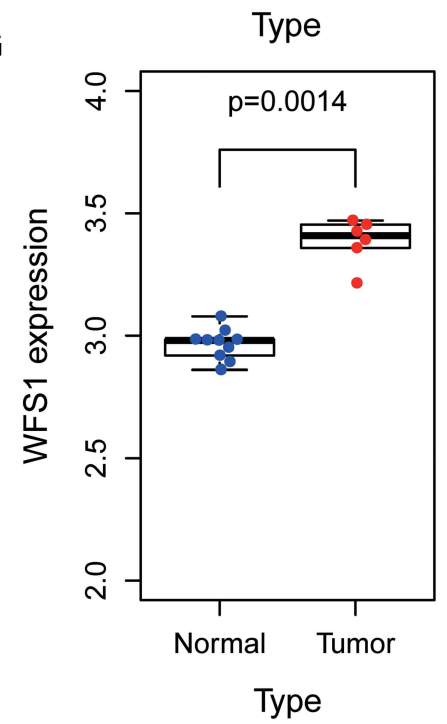

B

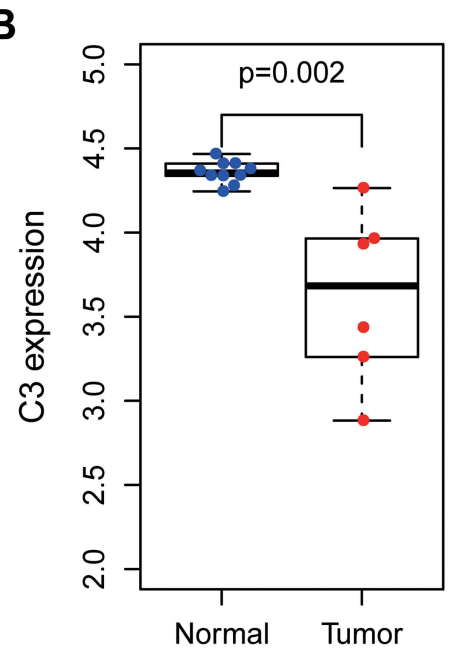

E

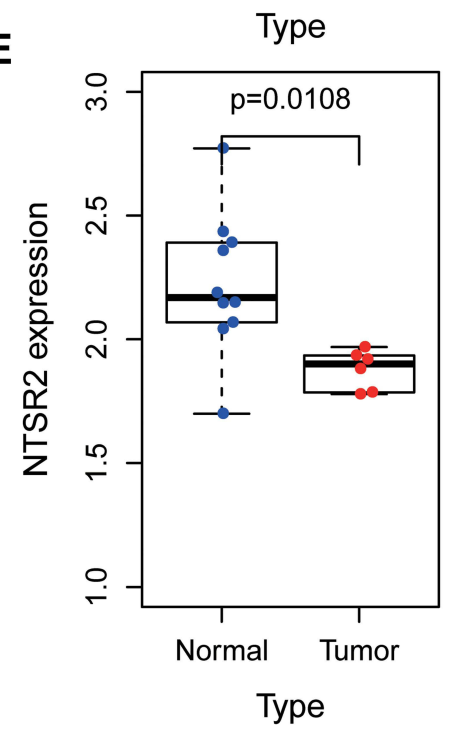

C

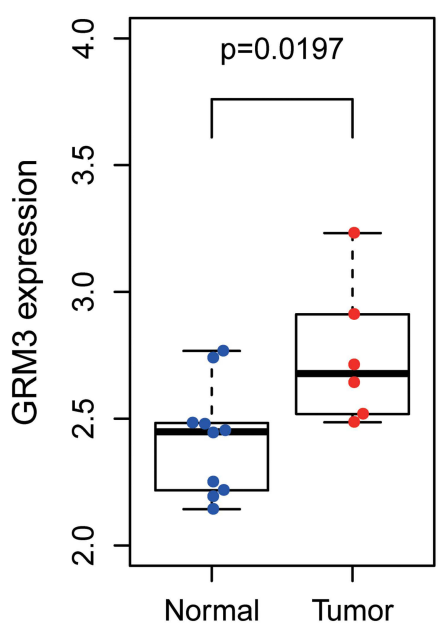

F

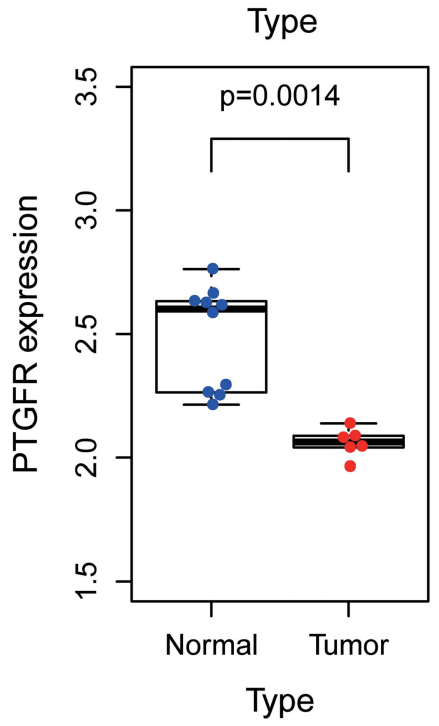

Figure 6 Validation of the expression levels of seven hub genes between APA tissues and normal adrenal tissues in GSEI0927. (A) AVPRIA. (B) C3. (C) GRM3. (D) JUN. (E) NTSR2. (F) PTGFR. (G) WFSI. 
A

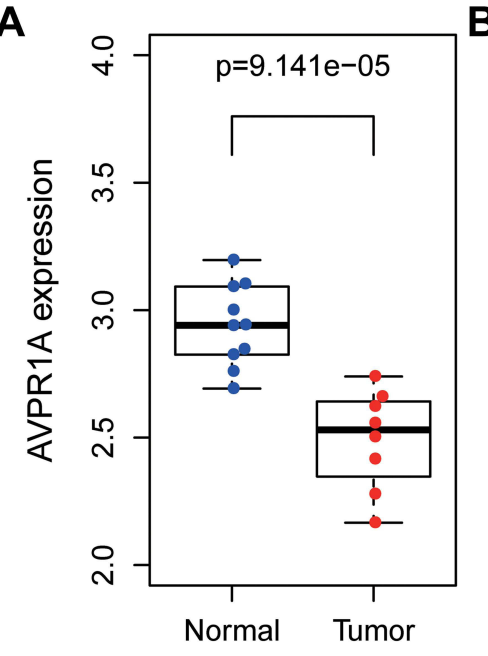

D

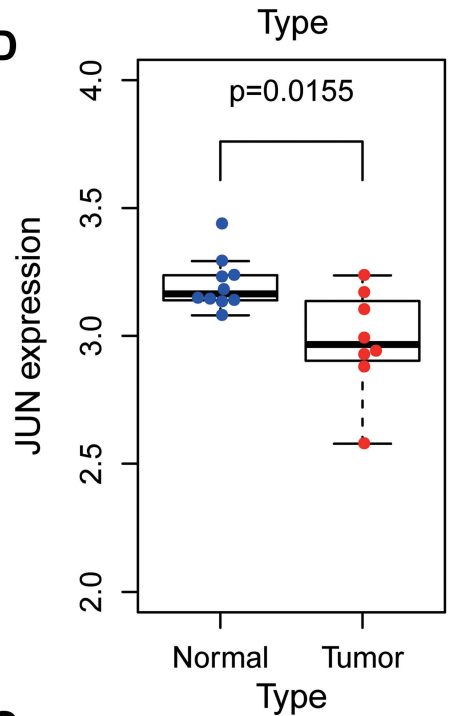

G

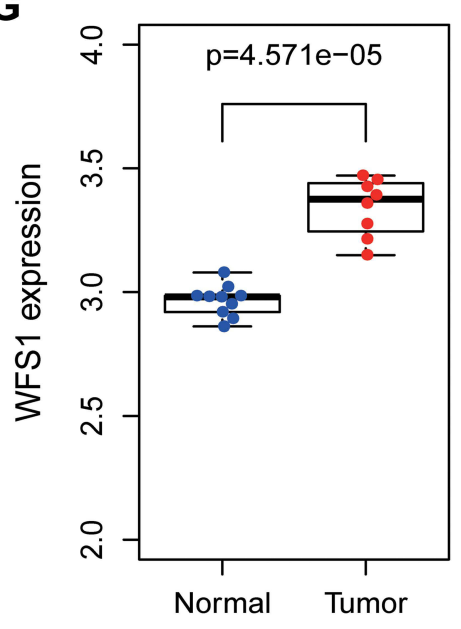

B

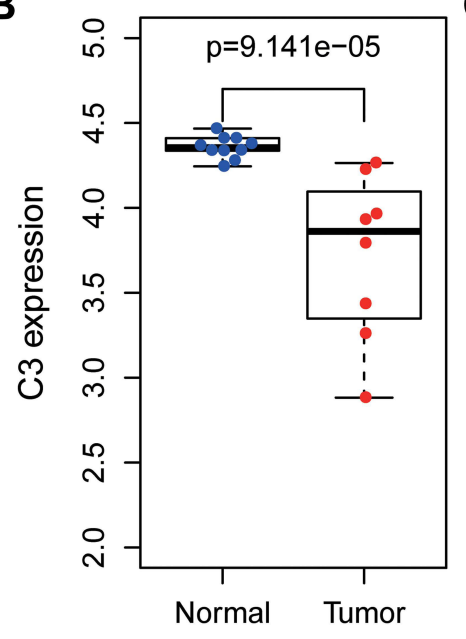

E

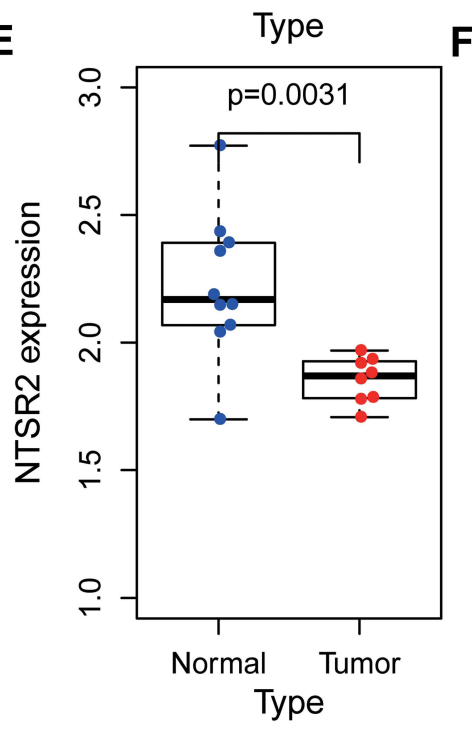

C

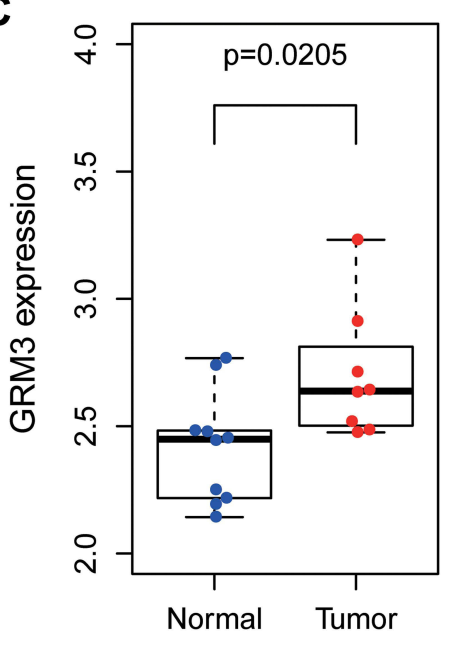

$\mathbf{F}$

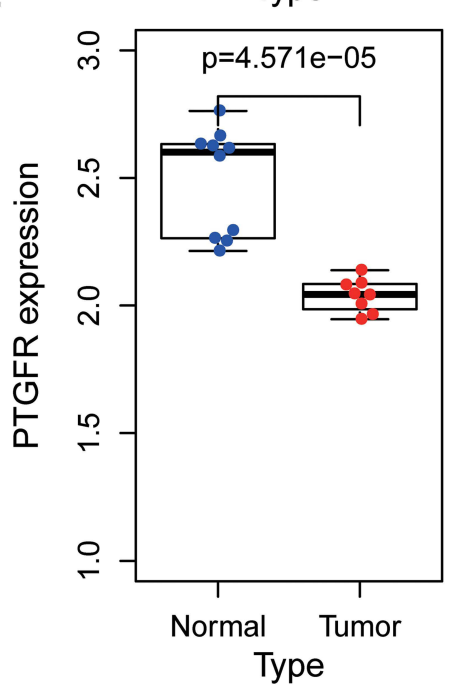

Type

Figure 7 Validation of the expression levels of seven hub genes between APA tissues and normal adrenal tissues in GSE3337I. (A) AVPRIA. (B) C3. (C) GRM3. (D) JUN. (E) NTSR2. (F) PTGFR. (G) WFSI. 
adrenal hyperplasia (BAH) and APA are considered as the most common causes of PA. According to the latest guidelines, unilateral adrenalectomy (UA) not only is the preferred treatment for APA but also has been shown to be effective in preventing adverse consequences of PA. Nevertheless, the assessment of postoperative benefit of $\mathrm{UA}$ is still difficult, and the investigation of prognostic factors is processing continuously. ${ }^{14}$

In this study, comprehensive bioinformatics approaches were used to identify genetic variance between APA and normal adrenal gland. The gene co-expression network was generated; PPI network analyses were performed, and key hub genes were highlighted. Interestingly, we successfully filtrated several genes, which might be associated with the development and progression of APA. GO term enrichment analysis revealed that, during the development of APA, cellular divalent inorganic cation homeostasis, calcium ion homeostasis, collagen-containing extracellular matrix, transport vesicle, metal ion transmembrane transporter activity and monovalent inorganic cation transmembrane transporter activity played essential roles. KEGG pathway analysis revealed that neuroactive ligand-receptor interaction, calcium signaling pathway, adrenergic signaling in cardiomyocytes, phagosome, and hematopoietic cell lineage might be crucial in APA. The alterations in calcium ion homeostasis, an important component of tumor microenvironment, is considered as hallmarks of carcinoma. ${ }^{15}$ These hallmarks include evasion of apoptosis, invasion and metastasis, unlimited replication, insensitivity to antigrowth signals and sustained angiogenesis. ${ }^{16-18}$

From GSE60042 and GSE8514, we identified 11 candidate genes. Further validation in two independent datasets revealed that there are seven hub genes involving in the development and progression of PA and APA, including C3, GRM3, AVPR1A, WFS1, PTGFR, NTSR2, and JUN. Felizola et $\mathrm{al}^{19}$ reported that the level of GRM3 mRNA was higher in APA than that in cortisol-producing adenoma (CPA) or normal adrenal cortex (NAC) and considered that GRM3 regulates steroidogenesis in adrenocortical tissues. Kiyotaka et $\mathrm{al}^{20}$ revealed that, in microarray, GRM3 was highly expressed in APA; however, in qPCR analysis, there are no different expressions in APA. The difference between the two methods requires further research to confirm. Zhao et $\mathrm{al}^{21,22}$ revealed that AVPR1A played important roles in CRPC progression and metastasis. Akiyama et $\mathrm{al}^{23}$ suggested that PTGFR is a novel marker for tumor endothelial cells (TECs) in renal cell carcinoma. Stockwin et $\mathrm{al}^{24}$ demonstrated that NTSR2 was involved in the growth and invasion in alveolar soft-part sarcoma (ASPS). Ye et $\mathrm{al}^{25}$ demonstrated that $\mathrm{C} 3$ is correlated to gastric cancer prognosis. JUN is a well-known oncogene and has been reported to be involved in various tumors, such as breast cancer, ${ }^{26,27}$ renal cell carcinoma, ${ }^{28}$ and ovarian cancer. ${ }^{29}$ The relationship between WFS1 expression and cancer development has not been fully illuminated yet. Recent studies have revealed that WFS1 might have associations with diabetes. ${ }^{30,31}$ Metabolic reprogramming has gradually reported to be a potential and vital mechanism for cancer $^{32,33}$ and diabetes could increase cancer progression. ${ }^{34}$ But little is aware of how high glucose may intrigue the genetic alterations resulting in tumor phenotypes. Hence, WFS1 might be a hub gene for APA metabolic reprogramming. In conclusion of all the results above, they indicated that above mentioned 7 hub genes might be potential biomarkers in the development of APA and PA. However, we have to admit the limitations of this study. Firstly, our sample size may be too small to observe some connections. More study with large volume of candidates is necessary. Secondly, experimental evidences are still missing. It can be more persuasive if we further validate our findings through IHC.

\section{Strengths and Limitations of This Study}

We comprehensively analyze hub genes through bioinformatics method.

These hub genes, which may involve in the development and progression of APA and PA, possibly act as potential biomarkers and novel therapeutic targets for APA and PA.

Further studies and experiments are still required to provide solid foundation of the function of the identified genes and pathways in vitro and in vivo.

\section{Conclusions}

To summarize, we comprehensively analyze hub genes through bioinformatics method. These hub genes, which may involve in the development and progression of APA and PA, possibly act as potential biomarkers and novel therapeutic targets for APA and PA. Nevertheless, to be cautious, further studies and experiments are still required 
to provide solid foundation of the function of the identified genes and pathways in vitro and in vivo.

\section{Data Sharing Statement}

All data generated or analyzed during the present study was downloaded from GEO database.

\section{Ethics Approval and Consent to Participate}

This study was approved by the Ethics Committee of the First Affiliated Hospital of Fujian Medical University.

\section{Author Contributions}

All authors made a significant contribution to the work reported, whether that is in the conception, study design, execution, acquisition of data, analysis and interpretation, or in all these areas; took part in drafting, revising or critically reviewing the article; gave final approval of the version to be published; have agreed on the journal to which the article has been submitted; and agree to be accountable for all aspects of the work.

\section{Funding}

There is no funding to report.

\section{Disclosure}

All authors declare no conflicts of interest.

\section{References}

1. Rossi GP. A comprehensive review of the clinical aspects of primary aldosteronism. Nat Rev Endocrinol. 2011;7(8):485-495. doi:10.1038/ nrendo.2011.76

2. Funder JW, Carey RM, Mantero F, et al. The management of primary aldosteronism: case detection, diagnosis, and treatment: an endocrine society clinical practice guideline. $J$ Clin Endocrinol Metab. 2016;101:1889-1916. doi:10.1210/jc.2015-4061

3. Rossi GP, Sacchetto A, Visentin P, et al. Changes in left ventricular anatomy and function in hypertension and primary aldosteronism. Hypertension. 1996;27:1039-1045. doi:10.1161/01.HYP.27.5.1039

4. Yang Q, Wang R, Wei B, et al. Candidate biomarkers and molecular mechanism investigation for glioblastoma multiforme utilizing WGCNA. Biomed Res Int. 2018;2018:4246703. doi:10.1155/2018/4246703

5. Yin L, Cai Z, Zhu B, Xu C. Identification of key pathways and genes in the dynamic progression of HCC based on WGCNA. Genes. 2018;9 (2):92. doi:10.3390/genes 9020092

6. Di Y, Chen D, Yu W, Yan L. Bladder cancer stage-associated hub genes revealed by WGCNA coexpression network analysis. Hereditas. 2019;156:7. doi:10.1186/s41065-019-0083-y

7. Tang J, Kong D, Qiuxia C, et al. Prognostic genes of breast cancer identified by gene co-expression network analysis. Front Oncol. 2018;8:374. doi:10.3389/fonc.2018.00374

8. Xia WX, Yu Q, Li G-H, et al. Identification of four hub genes associated with adrenocortical carcinoma progression by WGCNA. PeerJ. 2019;7:e6555. doi:10.7717/peerj.6555
9. Murakami M, Yoshimoto T, Nakabayashi K, et al. Integration of transcriptome and methylome analysis of aldosterone-producing adenomas. Eur $J$ Endocrinol. 2015;173(2):185-195. PMID: 25953827. doi:10.1530/EJE-15-0148

10. Ye P, Mariniello B, Mantero F, et al. G-protein-coupled receptors in aldosterone-producing adenomas: a potential cause of hyperaldosteronism. $J$ Endocrinol. 2007;195(1):39-48. PMID: 17911395. doi:10.1677/JOE-07-0037

11. Giordano TJ, Kuick R, Else T, et al. Molecular classification and prognostication of adrenocortical tumors by transcriptome profiling. Clin Cancer Res. 2009;15(2):668-676. PMID: 19147773. doi:10.1158/1078-0432.CCR-08-1067

12. Heaton JH, Wood MA, Kim AC, et al. Progression to adrenocortical tumorigenesis in mice and humans through insulin-like growth factor 2 and $\beta$-catenin. Am J Pathol. 2012;181(3):1017-1033. PMID: 22800756. doi:10.1016/j.ajpath.2012.05.026

13. Kaplan NM. The current epidemic of primary aldosteronism: causes and consequences. J Hypertens. 2004;22:863-869. doi:10.1097/ 00004872-200405000-00001

14. Kanarek-Kucner J, Stefański A, Barraclough R, et al. Insufficiency of the zona glomerulosa of the adrenal cortex and progressive kidney insufficiency following unilateral adrenalectomy - case report and discussion. Blood Press. 2018;27(5):304-312. doi:10.1080/ 08037051.2018.1470460

15. Tajbakhsh A, Pasdar A, Rezaee M, et al. The current status and perspectives regarding the clinical implication of intracellular calcium in breast cancer. J Cell Physiol. 2018;233(8):5623-5641. doi: $10.1002 /$ jcp. 26277

16. Prevarskaya N, Skryma R, Shuba Y. Ion channels and the hallmarks of cancer. T Rends Mol Med. 2010;16:107-121. doi:10.1016/j. molmed.2010.01.005

17. Hanahan D, Weinberg RA. The hallmarks of cancer. Cell. 2000;100 (5):7-70. doi:10.1016/S0092-8674(00)81683-9

18. Bose T, Cieślar-Pobuda A, Wiechec E, et al. Role of ion channels in regulating $\mathrm{Ca} 2+$ homeostasis during the interplay between immune and cancer cells. Cell Death Dis. 2015;6(2):e1648. doi:10.1038/ cddis. 2015.23

19. Felizola SJA, Nakamura Y, Satoh F, et al. Glutamate receptors and the regulation of steroidogenesis in the human adrenal gland: the metabotropic pathway. Mol Cell Endocrinol. 2014;382(1):170-177. doi:10.1016/j.mce.2013.09.025

20. Itcho K, Oki K, Kobuke K, et al. Aberrant G protein-receptor expression is associated with DNA methylation in aldosterone-producing adenoma.Mol. Cell Endocrinol. 2018;461:100-104. doi:10.1016/j. mce.2017.08.019

21. Zhao N, Peacock SO, Lo CH, et al. Arginine vasopressin receptor 1a is a therapeutic target for castration-resistant prostate cancer. Sci Transl Med. 2019;11:498. doi:10.1126/scitranslmed.aaw4636

22. Fenner A. AVPR1A: a target in CRPC? Nat Rev Urol. 2019;16 (9):508. doi:10.1038/s41585-019-0218-y

23. Akiyama K, Ohga N, Maishi N, et al. The F-prostaglandin receptor is a novel marker for tumor endothelial cells in renal cell carcinoma. Pathol Int. 2013;63(1):37-44. doi:10.1111/pin.12031

24. Stockwin LH, Vistica DT, Kenney S, et al. Gene expression profiling of alveolar soft-part sarcoma (ASPS). BMC Cancer. 2009;9. doi:10.1186/1471-2407-9-22

25. Ye J, Ren Y, Chen J, et al. Prognostic significance of preoperative and postoperative complement $\mathrm{C} 3$ depletion in gastric cancer: a three-year survival investigation. Biomed Res Int. 2017:2017. doi: $10.1155 / 2017 / 2161840$

26. Vogt PK. Jun, the oncoprotein. Oncogene. 2001;20(19):2365-2377. doi:10.1038/sj.onc. 1204443

27. Vleugel MM, Greijer AE, Bos R, et al. c-Jun activation is associated with proliferation and angiogenesis in invasive breast cancer. Hum Pathol. 2006;37(6):668-674. doi:10.1016/j.humpath.20 06.01 .022 
28. Oya M, Mikami S, Mizuno R, et al. C-Jun activation in acquired cystic kidney disease and renal cell carcinoma. J Urol. 2005;174 (2):726-730. doi:10.1097/01.ju.0000164656.99251.77

29. Eckhoff K, Flurschütz R, Trillsch F, et al. The prognostic significance of Jun transcription factors in ovarian cancer. $J$ Cancer Res Clin Oncol. 2013;139(10):1673-1680. doi:10.1007/s00432-013-1489-y

30. Zalloua PA, Azar ST, Delépine M, et al. WFS1 mutations are frequent monogenic causes of juvenile-onset diabetes mellitus in Lebanon. Hum Mol Genet. 2008;17(24). doi:10.1093/hmg/ddn304

31. Sharaf SA, Kantoush NA, Ayoub DF, et al. Altered expression of WFS1 and NOTCH2 genes associated with diabetic nephropathy in T2DM patients. Diabetes Res Clin Pract. 2018:140. doi:10.1016/j. diabres.2018.03.053
32. Biswas SK. Metabolic reprogramming of immune cells in cancer progression. Immunity. 2015;43:435-449. doi:10.1016/j.immuni.2 015.09.001

33. Weiss RH. Metabolomics and metabolic reprogramming in kidney cancer. Semin Nephrol. 2018;38:175-182. doi:10.1016/j.semne phrol.2018.01.006

34. Giovannucci E, Harlan DM, Archer MC, et al. Diabetes and cancer: a consensus report. CA Cancer J Clin. 2010;60:207-221. doi:10.3 322/caac. 20078

\section{Publish your work in this journal}

The International Journal of General Medicine is an international, peer-reviewed open-access journal that focuses on general and internal medicine, pathogenesis, epidemiology, diagnosis, monitoring and treatment protocols. The journal is characterized by the rapid reporting of reviews, original research and clinical studies across all disease areas. The manuscript management system is completely online and includes a very quick and fair peer-review system, which is all easy to use. Visit http://www.dovepress.com/ testimonials.php to read real quotes from published authors.

Submit your manuscript here: https://www.dovepress.com/international-journal-of-general-medicine-journal 